\title{
DERIVING PRIORITIES FROM PARTIALLY-FILLED RECIPROCAL COMPARISON MATRICES
}

\author{
Ami Arbel \\ Tel Aviv University, Tel Aviv 69978, Israel \\ ami@eng.tau.ac.il \\ Luis G. Vargas \\ The Joseph M Katz Graduate School of Business \\ University of Pittsburgh, Pittsburgh, PA 15260 USA \\ lgvargas@pitt.edu
}

\begin{abstract}
The question of centers addresses the issue of how to inscribe an object within a region defined by a set of constraints. More than one centering approach can be defined which leads to a different inscribed object and a different derivation procedure for both the object as well as its center. When attempting to inscribe the largest sphere within the constraints polytope the problem is defined as one of finding the Euclidean center of that polytope. We address in this paper various issues associated with the derivation of the Euclidean center and illustrate one application of this center to interval reciprocal matrices.
\end{abstract}

KEYWORDS: Euclidean Centers; Linear programming; Interval Reciprocal matrices

\section{Introduction}

Constrained optimization problems are defined by a set of constraints defining a region in space. The specific region is, of course, affected by the nature of the constraints. The latter may be either linear or nonlinear, but the question of centers applies to both. Essentially, this question addresses the issue of how to inscribe an object within the region defined by the constraints. More than one centering approach can be defined which leads to a different inscribed object and a different derivation procedure for both the inscribed object as well as its center. In this paper we focus our main attention on the Euclidean center, as we find it the most suitable center for Multiple-Objective Linear Programming (MOLP) problems.

The concept of a center has been a problem of some interest a few decades ago and has seen a rekindled interest in recent years. While it is important to many theoretical and applied problemsranging from location theory to interior-point linear programming algorithms-specific literature on the subject is quite scant. The first publication that has treated the question of centers in an explicit manner is that of Huard (1967). In this paper, he develops a general algorithm for optimizing a concave function over a convex feasible region with the use of centers and bounds. The generality of the algorithm is maintained through the general definition of the distance that was used. However, due to the general formulation considered in that paper, only necessary conditions for distance were mentioned. Sonnevend (1985) defined an analytic center and used it to develop a linear programming approach based on Karmarkar's interior-point projective algorithm (Karmarkar, 1984). Boggs et al. (1989) used Huard's method of centers to enhance interior-point methods using dual affine trajectories. Fagan and Falk (1996) introduced a method of Euclidean centers for solving single-objective linear programming problems. Their work uses Euclidean center without identifying its origin, which seems to defy an original source. In a recent book by G.B. Dantzig, the idea of a Euclidean center is mentioned as an exercise to the student 
(Dantzig and Thapa, 1997, Ex. 6.1, p.151). Parenthetically we add that the suggestion in the book will not lead to the proper center.

The above references treat the issue of centers in an explicit manner. This issue appears in a less explicit manner in other areas as well. Location theory, for example, has traditionally been interested in establishing the best location for placing a service node in a given network (see, e.g., Hansen et al, 1987). While not addressed as a centering problem specifically, such a problem is exactly that of finding a center. One may be interested in finding a point that is as close as possible to all nodes in the network or, conversely, finding a node that is as far away as possible from all nodes in a network (for disposing of waste, for example). Such problems measure distances from nodes that form a network.

As seen from the above survey, more than one center has been developed in the past and more than one application has been identified (Sadka, 1998). Limiting our discussion to linear systems of constraints simplifies the way we derive these centers. It does not, however, reduce the possibilities available for consideration. A center can be defined in more than one way and each way leads to different analytic and geometrical implications. Specifically, we note that the intersection of a set of linear constraints for a bounded linear programming problem defines a polytope in $n$-dimensional space. When one talks about a center of such a polytope one usually refers to the point from which one inscribes some object contained by the polytope. The reasoning behind this operation, as well as the type of object used-be it a sphere, or an ellipsoid, for example - leads to different definitions for a center. When one wishes to be as far as possible from all facets of the polytope - which is equivalent to inscribing the largest sphere - we derive the Euclidean center. Its derivation, properties and MOLP applications are the topics of this paper.

This paper is arranged as follows. In Section 2 we define a distance between a vector and a hyperplane in $n$-dimensional space and develop a formula for its calculation. We proceed in Section 3 to introduce the notion of a Euclidean center, including a discussion of computational methods for finding it, its geometrical interpretation and some of its special properties. In Section 4 we suggest an applications of the Euclidean center for MOLP problems. Section 5 provides summary and concluding remarks.

\section{The Euclidean Center}

The Euclidean center of a polytope is defined as the center of the largest inscribed sphere. The use of the Euclidean center is most common in facility location theory, for example, where one seeks the best location for a so-called obnoxious facility. The resulting solution provides a location, which is most distant from every inhabitant site in the area.

Denoting the interior space of an $n$-dimensional polytope with $m$ facets as $\boldsymbol{S}$, a vector $\boldsymbol{x} \in \mathrm{R}^{n}$ is inside the polytope when $\boldsymbol{x} \in \boldsymbol{S}$. Assuming that the distance between $\boldsymbol{x}$ and the $i$-th facet, where $i=1, \ldots, m$, is $d_{i}(\boldsymbol{x})$, the Euclidean center of this polytope is found by solving

$$
\underset{\boldsymbol{x} \in \boldsymbol{S}}{\operatorname{Max}}\left\{\underset{1 \leq i \leq m}{\operatorname{Min}}\left\{d_{\mathbf{i}}(\boldsymbol{x})\right\}\right\}
$$

The formulation in (2.1) maximizes - over the feasible set-the minimal distance to all the constraints. The latter include inequality constraints as well as the usual nonnegativity constraints. Next, we develop the defining equations for the feasible set.

A general linear programming problem is defined through 
$\max c^{T} \boldsymbol{x}$

$$
\begin{array}{ll}
\text { s.t. } & \mathbf{A}_{0} \boldsymbol{x}=\boldsymbol{b}_{0} \\
& \mathbf{A}_{1} \boldsymbol{x} \leq \boldsymbol{b}_{1} \\
& \mathbf{A}_{2} \boldsymbol{x} \geq \boldsymbol{b}_{2} \\
& \boldsymbol{x} \geq \boldsymbol{O}, \boldsymbol{x} \in \mathrm{R}^{n}, \boldsymbol{b}_{0} \in \mathrm{R}^{m_{0}}, \boldsymbol{b}_{1} \in \mathrm{R}^{m_{1}}, \boldsymbol{b}_{2} \in \mathrm{R}^{m_{2}},
\end{array}
$$

where $\mathbf{A}_{0} \in \mathbf{R}^{m_{0} \times n}, \mathbf{A}_{1} \in \mathbf{R}^{m_{1} \times n}$, and $\mathbf{A}_{2} \in \mathbf{R}^{m_{2} \times n}$. When converting the formulation in (2.2) to derive its Euclidean center, the equality constraints do not involve any distance measures. The inequality constraints, however, are converted to equality constraints by the usual slack and surplus variables. The only difference from the usual case is that each slack/surplus variable is multiplied by the Euclidean norm of its respective row. Similarly, the nonnegativity constraints require $\mathrm{n}$ surplus variables whose coefficient is 1 as it is the Euclidean norm of each individual nonnegativity constraint $x_{i} \geq 0$. Specifically, each of the inequality constraints are modified as followed:

Case 1: $\mathbf{A}_{1} x \leq b_{1}$

Denoting the $i$-th row of $\mathbf{A}_{1}$ by $\mathbf{A}_{1}^{i}$, the conversion to equality constraints is accomplished through

$$
\mathbf{A}_{1}^{i} \boldsymbol{x}+\left\|\mathbf{A}_{1}^{i}\right\|_{2} d_{i}=b_{1}^{i}, \forall 1 \leq i \leq m_{1}
$$

where $b_{1}^{i}$ is the $i$-th component of the vector $\boldsymbol{b}_{1}$., and $\left\|\mathbf{A}_{1}^{i}\right\|_{2}$ is the Euclidean norm of $\mathbf{A}_{1}^{i}$.

Case 2: $\mathbf{A}_{2} \boldsymbol{x} \geq \boldsymbol{b}_{2}$

Similarly, by denoting the $i$-th row of $\mathbf{A}_{2}$ by $\mathbf{A}_{2}^{i}$, the conversion to equality constraints is accomplished through

$$
\mathbf{A}_{2}^{i} \boldsymbol{x}-\left\|\mathbf{A}_{2}^{i}\right\|_{2} d_{i}=b_{2}^{i}, \forall 1 \leq i \leq m_{1}
$$

where $b_{2}^{i}$ is the $i$-th component of the vector $\boldsymbol{b}_{2}$.

Case 3: $x \geq 0$

The norm of each individual constraint is 1 and, therefore, these $n$ constraints are translated to

$$
x_{i}-d_{i}=0, \forall 1 \leq i \leq n
$$

\section{The Feasible Set}

The feasible set whose Euclidean center we seek is comprised of four components:

(a) The equality constraints: $\mathbf{A}_{0} \boldsymbol{x}=\boldsymbol{b}_{0}$,

(b) The inequality constraints: $\mathbf{A}_{1} \boldsymbol{x} \leq \boldsymbol{b}_{1}$ and $\mathbf{A}_{2} \boldsymbol{x} \geq \boldsymbol{b}_{2}$,

(c) The nonnegativity constraints on the solution vector, $\boldsymbol{x} \geq \boldsymbol{O}$,

Next, we build up the constraints set for deriving the Euclidean center. The equality constraints are not modified and appear as-is. Next, let's consider the inequality constraints for parts (a). Letting $\boldsymbol{d}_{1}$ and 
$\boldsymbol{d}_{2}$ represent the distance measure for each set of constraints in (a), respectively, results in the following system of linear equations.

$$
\left\{\begin{array}{l}
\mathbf{A}_{1} \boldsymbol{x} \leq \boldsymbol{b}_{1} \\
\mathbf{A}_{2} \boldsymbol{x} \geq \boldsymbol{b}_{2}
\end{array}, \quad \Rightarrow \quad\left\{\begin{array}{l}
\mathbf{A}_{1} \boldsymbol{x}+\mathbf{D}_{1} \boldsymbol{d}_{1}=\boldsymbol{b}_{1} \\
\mathbf{A}_{2} \boldsymbol{x}-\mathbf{D}_{2} \boldsymbol{d}_{2}=\boldsymbol{b}_{2}
\end{array}\right.\right.
$$

Next, considering the nonnegativity constraints and noting that the Euclidean norm for each one of these constraints is simply one, we arrive at the following

$$
\boldsymbol{x} \geq \boldsymbol{O}, \quad \Rightarrow \quad \boldsymbol{x}-\boldsymbol{d}_{0}=0
$$

where $\boldsymbol{d}_{0}$ represents the distance to the nonnegativity constraints.

The constraint in Error! Reference source not found.-Error! Reference source not found. are now augmented to form the system of equality constraints shown below

$$
\hat{\mathbf{A}} \hat{\boldsymbol{x}}=\hat{\boldsymbol{b}}, \quad \text { where }: \hat{\mathbf{A}}=\left(\begin{array}{c:c:c:c}
\mathbf{A}_{0} & \mathbf{0} & \mathbf{0} & \mathbf{0} \\
\hdashline \mathbf{A}_{1} & \mathbf{D}_{1} & \mathbf{0} & \mathbf{0} \\
\hdashline \mathbf{A}_{2} & \mathbf{O} & -\mathbf{D}_{2} & \mathbf{0} \\
\hdashline \mathbf{I}_{n}^{-} & \mathbf{O} & \mathbf{0} & -\mathbf{I}_{n}
\end{array}\right), \quad \hat{\boldsymbol{b}}=\left(\begin{array}{l}
\boldsymbol{b}_{0} \\
\frac{\boldsymbol{b}_{1}}{} \\
\frac{\boldsymbol{b}_{2}}{\boldsymbol{0}}
\end{array}\right)
$$

The matrices $\mathbf{D}_{1}$ and $\mathbf{D}_{2}$ are, respectively, $m_{1} \times m_{1}$ and $m_{2} \times m_{2}$ diagonal matrices whose diagonal elements are the Euclidean norms of the corresponding rows in the matrices $\mathbf{A}_{1}$ and $\mathbf{A}_{2}$. The augmented solution vector, $\hat{\boldsymbol{x}}$, is

$$
\hat{\boldsymbol{x}}=\left[\begin{array}{llll}
\boldsymbol{x} & \boldsymbol{d}_{1} & \boldsymbol{d}_{2} & \boldsymbol{d}_{0}
\end{array}\right]^{T}
$$

Where:

$\boldsymbol{x}$-is the original solution vector in (2.2),

$\boldsymbol{d}_{1}$ - The distance to each of the constraints of $\mathbf{A}_{1} \boldsymbol{x} \leq \boldsymbol{b}_{1}$,

$\boldsymbol{d}_{2}$ - The distance to each of the constraints of $\mathbf{A}_{2} \boldsymbol{x} \geq \boldsymbol{b}_{2}$,

$\boldsymbol{d}_{0}$ - The distance to the nonnegativity constraints $\boldsymbol{x} \geq \boldsymbol{O}$.

Using (2.8), the solution set, $\boldsymbol{S}$, is defined through

$$
S \triangleq\{\hat{\boldsymbol{x}} \mid \hat{\mathbf{A}} \hat{\boldsymbol{x}}=\hat{\boldsymbol{b}}\}
$$

\section{Finding the Euclidean Center}

To find the Euclidean center for the solution set $\boldsymbol{S}$ of (2.10) one has to solve a maxmin problem. Denoting the distance between a point in $\boldsymbol{S}$ and its $i$-th facet by $d_{i}(\boldsymbol{x})$, the Euclidean center of the solution set $\boldsymbol{S}$ is found by solving

$$
\underset{x \in S}{\operatorname{Max}}\left\{\operatorname{Min}_{i}\left\{d_{i}(\boldsymbol{x})\right\}\right\}
$$

Solving this maxmin problem is accomplished by considering first the "min" part and then the "max" 
part. The former is handled by defining an auxiliary variable, $\alpha$, which should be smaller than all the distance measures $d_{i}(\boldsymbol{x})$. That is: $\alpha \leq d_{1}^{i}, \alpha \leq d_{2}^{i}$ and $\alpha \leq d_{0}^{i}$, where $\boldsymbol{d}_{1}$ and $\boldsymbol{d}_{2}$ are the distance measure to the inequality constraints in Error! Reference source not found., and $\boldsymbol{d}_{0}$ is the distance measure to the nonnegativity constraints, $\boldsymbol{x} \geq \boldsymbol{O}$.

Together with the definition of $\boldsymbol{S}$, derived in (2.8), the resulting problem is now given by

$$
\begin{array}{lll} 
& \max \alpha & \\
\text { s.t. } & \alpha \leq d_{1}^{i} & \forall 1 \leq i \leq m_{1} \\
& \alpha \leq d_{2}^{i} & \forall 1 \leq i \leq m_{2} \\
& \alpha \leq d_{0}^{i} & \forall 1 \leq i \leq n \\
& \hat{\mathbf{A}} \hat{\boldsymbol{x}}=\hat{\boldsymbol{b}} & \boldsymbol{d}_{1}, \boldsymbol{d}_{2}, \boldsymbol{d}_{0} \geq \boldsymbol{O}
\end{array}
$$

Recalling (2.8), this problem is written compactly as

$$
\begin{aligned}
& \max \overline{\boldsymbol{c}}^{T} \overline{\boldsymbol{x}} \\
& \text { s.t. } \overline{\mathbf{A}} \overline{\boldsymbol{x}}=\overline{\boldsymbol{b}}
\end{aligned}
$$

where:

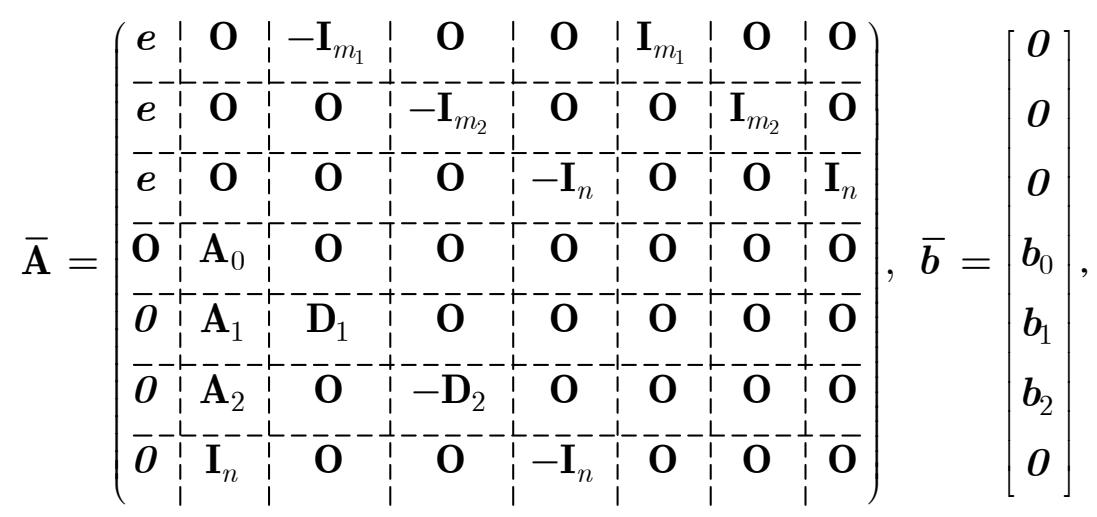

Notes concerning the solution to (2.13)-(2.14):

1. The constraints that are active in determining the inscribed sphere, are those for which $d_{i}=\alpha$.

2. The first three row blocks in $\overline{\mathbf{A}}$ correspond to the formulation responsible for solving the 'min' part of the maxmin problem of (2.11).

3. The last three blocks of columns of $\overline{\mathbf{A}}$ in (2.14) correspond to slack variables needed to convert the three sets of inequality constraints introduced in order to convert the 'min' part into equality constraints.

4. The vectors $\boldsymbol{e}$ and $\boldsymbol{0}$ in (2.14) are the unit and zero vectors, respectively.

5. The objective vector, $\overline{\boldsymbol{c}}$, is given by

$$
\overline{\boldsymbol{c}}=\left[\begin{array}{llll}
1 & 0 & \cdots & 0
\end{array}\right]^{T}, \text { where } \overline{\boldsymbol{c}} \in \mathbf{R}^{1+3 n+2\left(m_{1}+m_{2}\right)} .
$$

6. The solution vector, $\overline{\boldsymbol{x}}$, is defined by 


$$
\overline{\boldsymbol{x}}=\left[\begin{array}{llllllll}
\alpha & \boldsymbol{x} & \boldsymbol{d}_{1} & \boldsymbol{d}_{2} & \boldsymbol{d}_{0} & \boldsymbol{s}_{1} & \boldsymbol{s}_{2} & \boldsymbol{s}_{3}
\end{array}\right]^{T}, \overline{\boldsymbol{x}} \in \mathbf{R}^{1+3 n+2\left(m_{1}+m_{2}\right)}
$$

Next, we illustrate the solution process through some examples.

\section{The Euclidean Center of an Interval Reciprocal Matrix}

Euclidean centers are particularly useful in the study of reciprocal matrices whose entries are intervals. An interval reciprocal matrix is given by

$$
I J(A)=\left(\begin{array}{ccccc}
1 & {\left[l_{12} ; u_{12}\right]} & {\left[l_{13} ; u_{13}\right]} & \ldots & {\left[l_{1 n} ; u_{1 n}\right]} \\
& 1 & {\left[l_{23} ; u_{23}\right]} & \ldots & {\left[l_{2 n} ; u_{2 n}\right]} \\
& & 1 & \ldots & . \\
& & & \ldots & . \\
& & & & 1
\end{array}\right)
$$

Thus, in comparing elements $i$ and $j$, preference $\left(a_{i j}\right)$ may be stated through an inequality such as: $l_{i j} \leq a_{i j} \leq u_{i j}$, where $l_{i j}$ and $u_{i j}$ represent the lower and upper bounds, respectively. Following the formulation outlined above, the MaxMin Euclidean center is given by the solution to the following LP problem:

$$
\begin{aligned}
& \text { Max } \alpha \\
& \text { s.t., } \\
& w_{i}-d_{i}=0, i=1,2, \ldots, n \\
& w_{i}-u_{i j} w_{j}+\sqrt{\left(1+u_{i j}^{2}\right)} d_{h}=0, i, j=1,2, \ldots, n, h=n+1, \ldots, n+\frac{n(n-1)}{2} \\
& w_{i}-l_{i j} w_{j}-\sqrt{\left(1+l_{i j}^{2}\right)} d_{k}=0, i, j=1,2, \ldots, n, k=n+\frac{n(n-1)}{2}+1, \ldots, n^{2} \\
& w_{1}+\cdots+w_{n}=1 \\
& \alpha-d_{i} \leq 0, i=1,2, \ldots, n^{2}
\end{aligned}
$$

Even though judgments are approximations, it is possible that decision makers have doubts about which exact judgment to use. This may be due to lack of information or knowledge of the subject matter. Thus, interval judgments could be used to represent those situations where uncertainty is created not by the environment but by the lack of information. In this case, we propose to use the Euclidean center of the matrix as the priority of the elements being compared. The most interesting situation takes place when people not only have information uncertainty but they also have incomplete judgments.

\section{Partially-Filled Comparison Matrices}

How to deal with incomplete reciprocal matrices has been studied since Harker (1987) published his paper. There are a number of approaches using traditional statistical measures (Ohya 2007; Gao et al. 2010), minimizing inconsistency (Shiraishi et al. 1998; Obata et al. 1999; Fedrizzi and Giove 2006; Bozoki et al. 2009; ), and graph theory (Benroider et al. 2010). In this paper we show how to use Euclidean centers to study partially filled interval reciprocal matrices.

An $n \times n$ comparison matrix has elements, $a_{i j}$, that try to estimate the ratio of $w_{i}$ to $w_{j}$, where $w_{i}$ and $w_{j}$ are the priorities of the i-th and j-th elements, respectively. We want to use the idea of Euclidean 


\section{A. Arbel and L.G. Vargas}

Center to estimate the priorities of partially-filled matrices, i.e., matrices that have some missing entries. Unlike previous papers in the subject, we do not try to estimate the missing pairwise comparisons. We estimate the priorities of the elements compared with the judgments provided. For example, consider a $4 \times 4$ comparison matrix whose first row elements are described through

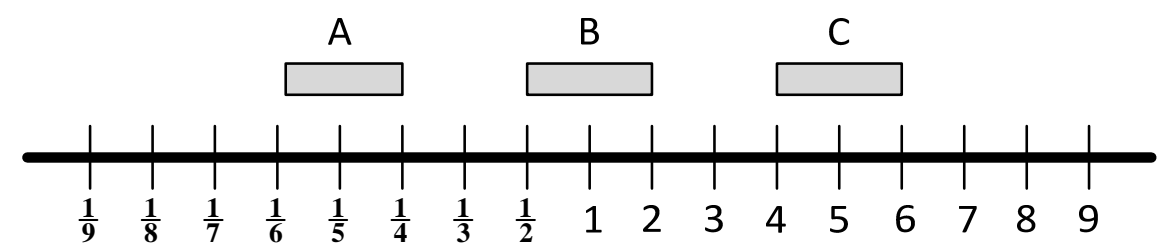

The region shown in region $\mathbf{A}$ corresponds to a pairwise comparison response of $\frac{1}{5} \pm 1$ scale unit, that of $\mathbf{B}$ to $1 \pm 1$ scale unit, and that for $\mathbf{C}$, corresponds to the interval of $5 \pm 1$ scale unit. These three responses form the first row to the resulting comparison matrix shown below:

$$
\mathbf{A}=\left[\begin{array}{cccc}
1 & {\left[\frac{1}{6}, \frac{1}{4}\right]} & {\left[\frac{1}{2}, 2\right]} & {[4,6]} \\
{[4,6]} & 1 & & \\
{\left[\frac{1}{2}, 2\right]} & & 1 & \\
{\left[\frac{1}{6}, \frac{1}{4}\right]} & & & 1
\end{array}\right],
$$

The Euclidean center of this matrix is given by $(0.1689,0.7039,0.0921,0.0351)^{\mathrm{T}}$. As the width of the interval around the judgments tends to zero the Euclidean center converges to $(0.1389,0.6944,0.1389$, $0.0278)^{\mathrm{T}}$ which is the vector $(1,5,1,1 / 5)^{\mathrm{T}}$ normalized using the $l_{1}$-norm. As long as the entries in the matrix form a spanning tree, the Euclidean center of the matrix will coincide with the principal right eigenvector of the consistent matrix derived from the spanning tree judgments. When the entries do not form a spanning tree, the problem may not have a solution because the feasible region may be empty. In Arbel and Vargas (2008) we showed that the Euclidean center exists if and only if $\alpha \geq 0$ in (3.1). For example, consider the matrix given by

$$
\mathbf{A}=\left[\begin{array}{cccc}
1 & {\left[\frac{1}{6}, \frac{1}{4}\right]} & & \\
& 1 & & {[2,4]} \\
& & 1 & \\
& & {[3,5]} & 1
\end{array}\right], \mathbf{W}=\left[\begin{array}{cccc}
1 & \frac{1}{5} & \frac{12}{5} & \frac{3}{5} \\
5 & 1 & 12 & 3 \\
\frac{5}{12} & \frac{1}{12} & 1 & \frac{1}{4} \\
\frac{5}{3} & \frac{1}{3} & 4 & 1
\end{array}\right]
$$

Because the entries of the matrix $\mathbf{A}$ form a spanning tree, the midpoints generate the consistent matrix $\mathbf{W}$. Thus, the Euclidean center and the PR-eigenvector coincide and the priorities are given by $(0.1237$, $0.6186,0.0515,0.2062)^{\mathrm{T}}$ as the width of the interval tends to zero. When the entries do not form a spanning tree, the priorities assigned to the alternatives that cannot be reached (or compared) will be equal to zero. If it is not a spanning tree because there are loops, but all the alternatives can be reached from all the others, then the Euclidean center will depend on the width of the interval selected. For example, if the matrix $\mathbf{A}$ is now given by

$$
\mathbf{A}=\left[\begin{array}{cccc}
1 & {\left[\frac{1}{6}, \frac{1}{4}\right]} & & \\
& 1 & {[1,3]} & {[2,4]} \\
& & 1 & \\
& & {[3,5]} & 1
\end{array}\right]
$$


but the MaxMin problem (3.1) does not always have a solution. For the matrix given above the smallest width of the interval for which there is a solution is equal to 1.5505. The new matrix $\mathbf{A}$ and corresponding Euclidean center are given by

$$
\mathbf{A}=\left[\begin{array}{cccc}
1 & {[0.153,0.289]} & & \\
& 1 & {[0.449,3.551]} & {[1.449,4.551]} \\
& & 1 &
\end{array}\right], \mathbf{w}=\left[\begin{array}{l}
0.0810 \\
0.4661 \\
0.1313 \\
0.3216
\end{array}\right]
$$

respectively. Note that we do not have an equivalent solution using the Eigenvector method.

\section{Summary}

We have shown that the Euclidean center of incomplete interval reciprocal matrices can be used to derive priorities when the eigenvector method cannot be used. We showed that when the entries of the incomplete matrix form a spanning tree the solution of the Euclidean center method coincide with that of the principal right eigenvector method. This approach can be used to provide judgments for the entries of the incomplete matrix of pairwise comparisons.

\section{REFERENCES}

Arbel, A. (1993), “An Interior Multiobjective Linear Programming Algorithm,” Computers Ops Res., Vol. 20, No. 7, pp. 723-735.

Arbel, A., Korhonen, P. (1996), "Using Aspiration Levels in an Interactive Interior Multiobjective Linear Programming Algorithm,” European Journal of Operational Research, Vol. 89, pp. 193-201.

Arbel, A., Vargas, L. (2008). “Euclidean Centers: Computation and a MOLP Application,” Mathematical and Computer Modeling 48, 1-2 (2008) 197-205.

Benroider, E. W. N., K. Maier and CV.Stix (2010). Incomplete Information within Relative Pairwise Comparisons as Utilized by the AHP. Business and Information Systems Workshops, LNBIP 57. R. T. W. Abramowicz, K. Wecel (eds.), Springer-Verlag: 39-50.

Boggs, P.T., Domich, P.D., Donaldson, J.R., and Witzgall, C. (1989), “Algorithmic Enhancements to the Method of Centers for Linear Programming Problems,” ORSA Journal on Computing, Vol. 1, No. 3, pp. 159-171.

Bozóki, S., Fülöp, J., Rónyai, L. [2009]: “Incomplete Pairwise Comparison Matrices in Multi-Attribute Decision Making," In: Proceedings of the IEEE International Conference on Industrial Engineering and Engineering Management (IEEM),Hong Kong, 8-11 Dec 2009, pp. 2256-2260., ISBN 978-14244-4870-8., DOI: 10.1109/IEEM.2009.5373064.

http://ieeexplore.ieee.org/stamp/stamp.jsp?tp=\&arnumber=5373064\&isnumber=5372873.

Dantzig, G.B., and Thapa M.N. (1997), Linear Programming 1: Introduction, Springer-Verlag.

Fagan, J.T., and Falk J.E. (1996), “A Method of Euclidean Centers,” in Computers Ops Res., Vol. 23, No. 1, pp. 13-25.

Fedrizzi, M. and S. Giove (2007). "Incomplete Pairwise Comparison and Consistency Optimization." European Journal of Operational Research 183(1): 303-313.

Gao, S., Z. Zhang and C. Cao (2010). "Calculating Weights Methods in Complete Matrices and Incomplete Matrices." Journal of Software 5(3): 304-311. 


\section{A. Arbel and L.G. Vargas}

Hansen, P., Labbe, M., Peeters, D., Thisse, J.F., and Henderson J.V. (1987), "Systems of Cities and Facility Location,” Fundamentals of Pure and Applied Economics 22.

Harker, P. (1987). "Incomplete Pairwise Comparisons in the Analytic Hierarchy Process. Mathematical and Computer Modeling 9: 838-848.

Huard, P. (1967), "Resolution of Mathematical Programming with Nonlinear Constraints by the Method of Centers,” in J. Abadie, (Ed.) Nonlinear Programming, North-Holland, pp. 209-219.

Karmarkar, N.K. (1984), “A New Polynomial Time Algorithm for Linear Programming,” Combinatorica 4, pp. 373-395.

Ohya, T. (2007). Investigating for Carrying Incomplete Pairwise Comparisons in AHP. ISAHP Proceedings. Viña Del Mar, Chile.

Rosen, J.B. (1960), “The Gradient Projection Method for Nonlinear Programming,” SIAM, Vol. 8, No. 1, pp.181-217.

Sadka, R. (1998), “Theory of Centers and its use in Multiple-Objective Linear Programming,” M.Sc. thesis, Tel-Aviv University, (unpublished manuscript).

Shiraishi, S., T. Obata and M. Daigo (1998). "Properties of a Positive Reciprocal Matrix and Their Application to AHP." Journal of the Operations Research Society of Japan 41(3): 404-414.

Steuer, R.E. (1986), Multiple Criteria Optimization: Theory, Computation and Application, Krieger.

Sonnevend, G. (1985), “An Analytical Center for Polyhedrons and New Classes of Global Algorithms for Linear (Smooth, Convex) Programming,” Proceedings $12^{\text {th }}$ IFIP Conference on System Modelling, Budapest. 